\title{
MicroRNA-100-5p inhibits osteoclastogenesis and bone resorption by regulating fibroblast growth factor 21
}

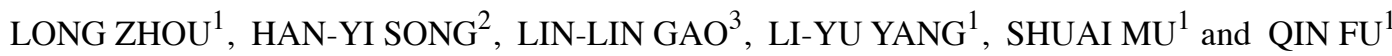 \\ Departments of ${ }^{1}$ Orthopedic Surgery and ${ }^{2}$ Gastroenterology; ${ }^{3}$ Animal Laboratory of Experimental Research Center, \\ Shengjing Hospital of China Medical University, Shenyang, Liaoning 110004, P.R. China
}

Received July 31, 2018; Accepted November 21, 2018

DOI: $10.3892 / \mathrm{ijmm} .2018 .4017$

\begin{abstract}
MicroRNAs (miRNAs/miRs) are post-transcriptional regulators that serve important roles in osteoclastogenesis and bone metabolism; however, the roles of miRNAs have not been completely clarified. The present study aimed to investigate the effects of miR-100-5p on the mechanism of liver-bone endocrine metabolism. A miRNA microarray analysis was conducted to evaluate the miRNA expression profile during receptor activator of nuclear factor- $\kappa \mathrm{B}$ ligand-stimulated osteoclastogenesis. Hematoxylin and eosin and tartrate-resistant acid phosphatase staining were performed to analyze the trabecular bone microstructure and osteoclast differentiation. The mRNA and protein expression levels were assessed by reverse transcription-quantitative polymerase chain reaction and western blotting, respectively. The results revealed that in vitro osteoclast differentiation and in vivo bone resorption were suppressed by miR-100-5p overexpression. In vivo, a decrease in miR-100-5p and an increase in FGF21 were simultaneously observed in mice following ovariectomy (OVX). Bioinformatics analysis and experimental data confirmed that FGF21 was a direct target of miR-100-5p. Conversely, augmentation of miR-100-5p using a specific agomir in OVX-operated mice decreased the levels of FGF21 in the serum and liver, and prevented osteoclastogenesis and bone loss. The present study revealed that FGF21 may be a signal molecule associated with the mechanism of liver-bone endocrine metabolism and may be targeted by miR-100-5p. In addition, miR-100-5p may serve an important role in protecting against OVX-induced osteoporosis.
\end{abstract}

Correspondence to: Dr Qin Fu, Department of Orthopedic Surgery, Shengjing Hospital of China Medical University, Floor 14, 2nd Building, 36 Sanhao Street, Heping, Shenyang, Liaoning 110004, P.R. China

E-mail: fu_qin022@163.com

Key words: microRNA-100-5p, fibroblast growth factor 21, ovariectomy, osteoporosis, osteoclastogenesis

\section{Introduction}

As a dynamic metabolic system, bone modeling or remodeling is modulated by two major bone cells: Osteoblasts, which are able to secrete bone matrix and accelerate calcium (Ca) deposition, and osteoclasts, which are responsible for resolving mineralized bone matrix (1-3). In the process of bone maintenance and repair, extracellular signaling transduction between osteoblasts and osteoclasts serves a crucial role in bone homeostasis (4). For example, osteoblast-secreted receptor activator of nuclear factor $-\kappa \mathrm{B}(\mathrm{NF}-\kappa \mathrm{B})(\mathrm{RANK})$ ligand (RANKL) binds to the RANK receptor on osteoclasts, thus promoting osteoclast survival and osteoclastogenesis (5). In addition, the binding of macrophage colony-stimulating factor (M-CSF) to its receptor, colony stimulating factor receptor, has been reported to be essential for the generation of osteoclast precursor cells that are present prior to RANKL stimulation (2). Furthermore, tumor necrosis factor receptor-associated factor-6-activated $\mathrm{NF}-\kappa \mathrm{B}$ signaling induces the initialization of nuclear factor of activated T cells, cytoplasmic 1 (NFATc1), which is a key transcription factor for osteoclastogenesis and stimulates the expression of various osteoclast-specific genes, including tartrate-resistant acid phosphatase (TRAP), cathepsin K (Ctsk) and calcitonin receptor $(2,5)$.

MicroRNAs (miRNAs/miRs) are a class of small, single-stranded, non-coding RNA molecules, 18-24 nucleotides in length, which can regulate gene expression or translation by targeting 3'-untranslated regions (3'-UTRs). miRNAs serve as a novel class of post-transcriptional regulators that are involved in diverse biological functions (6-8). Previous studies have reported that miRNAs serve important roles in regulating skeletal development $(9,10)$. For example, overexpression of miR-34a, miR-125a and miR-503 inhibits osteoclastogenesis to rescue bone loss $(9,11,12)$. Conversely, miR-214 promotes osteoclastogenesis, and inhibits osteoblast differentiation and bone formation $(10,13)$. miR-100 is a member of the miR-99 family (14) and participates in various biological processes, including chemotherapy resistance, apoptosis, osteogenic differentiation and osteoporotic fractures (15-18). However, the role of miR-100 in ovariectomy (OVX)-induced osteoporosis and osteoclastogenesis remains unclear.

Fibroblast growth factor (FGF)21 is a member of the FGF family, which is mainly expressed in the liver, and has numerous metabolic functions (19). FGF21 has been demonstrated to 
improve obesity-associated glucose and lipid metabolic disorders (20), renal dysfunction (21), cardiac hypertrophy (22) and ethanol-associated liver injury (23). Conversely, upregulation of FGF21 is an independent risk factor associated with the severity of non-alcoholic fatty liver disease (24). In a diet-induced mouse model of obesity, a reduction in plasma FGF21 levels and hepatic FGF21 resistance by exenatide protects against high-fat diet-induced non-alcoholic fatty liver disease (25). Emerging evidence has highlighted that FGF21 may serve as a potent regulator of skeletal homeostasis (26). In a Han Chinese population, a significant inverse correlation is observed between plasma FGF21 levels and bone mineral density (BMD) of the femoral neck and Ward's triangle (27). An increase in serum FGF21 levels is also negatively associated with BMD and bone mineral content in human immunodeficiency virus-1-infected patients (28). However, fasting plasma FGF21 levels in healthy women exhibit a strong positive association with total BMD and spine BMD (29). Notably, FGF21-knockout in mice results in a high-bone-mass phenotype, whereas FGF21 gain-of-function decreases bone mass and promotes osteoclastogenesis $(26,30)$. However, the underlying molecular mechanisms of FGF21-enhanced osteoclastogenesis and bone resorption have not been completely clarified.

The present study screened the expression profile of miRNAs in the process of M-CSF- and RANKL-induced osteoclastogenesis using a miRNA microarray. The results demonstrated that miR-100-5p was downregulated and functioned as a negative regulator of osteoclastogenesis. miR-100-5p gain-of-function significantly suppressed osteoclast activity in vitro. In vivo, treatment with the agomir-miR-100-5p inhibited FGF-21 expression and osteoclast activity, and prevented bone loss in mice following OVX.

\section{Materials and methods}

Cell culture. The present study was approved by the Animal Ethical Committee of Shengjing Hospital of China Medical University (Shenyang, China; approval no. 2016PS361K). Murine bone marrow-derived macrophages (BMMs) were obtained form female $\mathrm{C} 57 \mathrm{BL} / 6 \mathrm{~J}$ mice, as described previously (31). BMMs were maintained in $\alpha$-Minimum Essential Medium (HyClone; GE Healthcare Life Sciences, Logan, UT, USA) containing 10\% fetal bovine serum (FBS; Gibco; Thermo Fisher Scientific, Inc., Waltham, MA, USA) and $1 \%$ penicillin-streptomycin at $37^{\circ} \mathrm{C}$ in an atmosphere containing $5 \% \mathrm{CO}_{2}$. Non-adherent cells were cultured in M-CSF $(50 \mathrm{ng} / \mathrm{ml}$; R\&D Systems China Co., Ltd., Shanghai, China) for 3 days to induce osteoclast precursors. For osteoclast differentiation, osteoclast precursors were incubated with M-CSF (50 ng/ml) and RANKL (100 ng/ml; R\&D Systems China Co., Ltd.) for another 3 days (Fig. 1A). Osteoclasts with $\geq 3$ nuclei/cell were identified as TRAP-positive cells. The NCTC-1469 mouse normal liver cell line (serial number: 3111C0001CCC000290) was obtained from the Institute of Biochemistry and Cell Biology of the Chinese Academy of Sciences \& National Infrastructure of Cell Line Resource (Shanghai, China). The cells were cultured in Dulbecco's modified Eagle's medium (Gibco; Thermo Fisher Scientific, Inc.) supplemented with $5 \% \mathrm{FBS}$, in a humidified incubator containing $5 \% \mathrm{CO}_{2}$ and 95\% air (Thermo Fisher Scientific, Inc.).
Differentially expressed miRNAs in RANKL-induced osteoclast differentiation. Osteoclast differentiation was induced as shown in Fig. 1A. Subsequently, miRNA expression profiling of osteoclasts was conducted using the mouse miRNA expression profiling array V.2 (Illumina Inc., San Diego, CA, USA), according to the manufacturer's protocol. Hierarchical clustering was performed to determine similarity using complete linkage and Euclidean distance using $\mathrm{MeV}$ software (version 4.2.6; http://mev.tm4.org/\#/welcome) (32). The miRNAs with a fold-change of $>2$ in response to RANKL-stimulated osteoclast differentiation compared with precursor cells were selected to determine the expression profile.

Animal model. Female C57BL/6J mice (age, 8 weeks; body weight, $20 \pm 2 \mathrm{~g}$ ) were obtained from the Animal Center of Shengjing Hospital of China Medical University, and were allowed to acclimate for 1 week in a temperature-controlled environment $\left(25 \pm 2^{\circ} \mathrm{C}\right.$; humidity, $\left.55 \pm 5 \%\right)$ under an artificial 12-h light/dark cycle with free access to food and tap water. Mice were randomly divided into four groups ( $n=6 /$ group) and underwent either sham operation or bilateral OVX. Agomir-miR-100-5p (5'-AACCCGUAGAUCCGAACUUGU G-3') and control agomir (agomir-Con; 5'-AGUUUAGCU AACGGUGAGCCGA-3') were purchased from Guangzhou RiboBio Co. Ltd. Surgical operations were performed under sodium pentobarbital anesthesia $(50 \mathrm{mg} / \mathrm{kg}$; Sigma-Aldrich; Merck KGaA) by intraperitoneal injection. The sham group received the same surgical operation as the OVX group without ovariectomy, whereas mice in the OVX group the ovaries were excised. The groups were as follows: i) Sham group, sham-operated mice were treated with normal saline; ii) OVX group, OVX-operated mice were treated with normal saline; iii) OVX + agomir-Con group, OVX-operated mice were treated with agomir-Con; iv) OVX + agomir-miR group, OVX-operated mice were treated with agomir-miR-100-5p $(100 \mathrm{mg} / \mathrm{kg})$ via a tail vein injection. After a 4-week treatment with the agomirs (twice/week), mice were euthanized and blood was harvested from the heart. Serum, liver and tibia samples were immediately collected and maintained at $-80^{\circ} \mathrm{C}$ for further analysis.

Reverse transcription-quantitative polymerase chain reaction $(R T-q P C R)$. TotalRNA was extracted from RANKL-stimulated precursor cells, NCTC-1469 cells, and liver and tibia samples using TRIzol ${ }^{\circledR}$ (Invitrogen; Thermo Fisher Scientific, Inc.), according to the manufacturer's protocol. RT was performed using TaqMan ${ }^{\circledR}$ reverse transcription kit (Applied Biosystems; Thermo Fisher Scientific, Inc.), according to the manufacturer's protocol. miR-100-5p was detected using TaqMan ${ }^{\circledR}$ MicroRNA assay (Applied Biosystems; Thermo Fisher Scientific, Inc.), according to the manufacturer's protocol. The thermocycling conditions were as follows: $95^{\circ} \mathrm{C}$ for $10 \mathrm{~min}$, followed by 40 cycles at $95^{\circ} \mathrm{C}$ for $15 \mathrm{sec}$ and $60^{\circ} \mathrm{C}$ for $60 \mathrm{sec}$. U6 small nuclear RNA was used as an endogenous control. Relative miR-100-5p expression levels were calculated using the $2^{-\Delta \Delta \mathrm{Cq}}$ method (33).

In addition, $2 \mu \mathrm{g}$ total RNA was used to synthesize cDNA with moloney murine leukemia virus reverse transcriptase (Invitrogen; Thermo Fisher Scientific, Inc.), according to the 
Table I. Primers used for reverse transcription-quantitative polymerase chain reaction.

\begin{tabular}{lll}
\hline Gene & \multicolumn{1}{c}{ Forward primer (5'-3') } & Reverse primer (5'-3') \\
\hline NFATc1 & GCGAAGCCCAAGTCTCTTTCC & GTATGGACCAGAATGTGA \\
Ctsk & AGGCGGAGGTCGATGCCCCG & CACGATGATGTCACCCTCGATGT \\
MMP-9 & ACGCAGACATCGTCATCCAG & CAGGGACCACAACTCGTCAT \\
TRAP & GCTACTTGCGGTTTCACTATGGA & TGGTCATTTCTTGGGGCTTATCT \\
Runx2 & GGCAGGTGCTTCAGAACTGG & GTGGTGGCAGGTAGGTATGG \\
Osterix & TGAGCTGGAACGTCACGTGC & AAGAGGAGGCCAGCCAGACA \\
Osteocalcin & CTCAGGGTTTCAGTGGTT & TTTCCACGAGCACCCATC \\
ALP & CCCTCTCCAAGA CATATAACAC & TTGCCCTGAGTGGTGTTG \\
OPG & CACACACACTGGGGACTCTG & CAGCTGTGAGGAGAGGAAGG \\
RANKL & CACAGCCCTCTCTCTTGAGC & GACTGTGACCCCCTTCCATA \\
CA2 & CATTACTGTCAGCAGCGAGCA & GACGCCAGTTGTCCACCATC \\
FGF21 & CAGGGAGGATGGAACAGTGGTA & TGACACCCAGGATTTGAATGAC \\
GAPDH & CACCATGGAGAAGGCCGGGG & GACGGACACATTGGGGGTAG
\end{tabular}

ALP, alkaline phosphatase; CA2, carbonic anhydrase 2; Ctsk, cathepsin K; FGF21, fibroblast growth factor 21; MMP-9, matrix metalloproteinase-9; NFATc1, nuclear factor of activated T cells, cytoplasmic 1; OPG, osteoprotegrin; RANKL, receptor activator of nuclear factor- $\mathrm{\kappa B}$ ligand; Runx2, Runt-related transcription factor 2.

manufacturer's protocol. RT-qPCR was performed using the Applied Biosystems 7300 Real-Time PCR system (Applied Biosystems; Thermo Fisher Scientific, Inc.) with the TaqMan ${ }^{\circledR}$ Universal PCR Master Mix (Thermo Fisher Scientific, Inc.). The cycling conditions were as follows: $95^{\circ} \mathrm{C}$ for $10 \mathrm{~min}$, followed by 40 cycles at $95^{\circ} \mathrm{C}$ for $15 \mathrm{sec}, 60^{\circ} \mathrm{C}$ for $30 \mathrm{sec}$ and $72^{\circ} \mathrm{C}$ for $30 \mathrm{sec}$, and a final extension step at $72^{\circ} \mathrm{C}$ for $3 \mathrm{~min}$. The relative mRNA expression levels were calculated using the $2^{-\Delta \Delta \mathrm{Cq}}$ method (33) and were normalized to GAPDH. The primers were synthesized by Invitrogen; Thermo Fisher Scientific, Inc. and sequences are presented in Table I.

Cell transfection and plasmid constructs. Pre-miR-control (Pre-miR-Con) and pre-miR-100-5p were synthesized by Guangzhou RiboBio Co., Ltd. (Guangzhou, China). Osteoclast precursors $\left(1 \times 10^{5}\right)$ were seeded in 6-well plates and transfected with pre-miR-Con (5'-AGUUUAGCUAACGGUGAGC CGA-3') or pre-miR-100-5p (5'-AACCCGUAGAUCCGAACU UGUG-3') using Lipofectamine ${ }^{\circledR} 2000$ (Invitrogen; Thermo Fisher Scientifc, Inc., Waltham, MA, USA) for $48 \mathrm{~h}$ at $37^{\circ} \mathrm{C}$ at final concentrations of $100 \mathrm{nM}$, according to the manufacturer's protocol.

TRAP staining. RANKL-stimulated precursor cells and tibia samples were fixed in $4 \%$ paraformaldehyde for $20 \mathrm{~min}$ and $24 \mathrm{~h}$, respectively, at room temperature. Tibia histological sections $(5 \mu \mathrm{m})$ were obtained following decalcification in 0.5 MEDTA (pH 8.0) and were embedded in paraffin, according to standard histological procedures. RANKL-stimulated precursor cells and tibia histological sections were stained for TRAP activity using a $0.1 \mathrm{M}$ acetate solution ( $\mathrm{pH}$ 5.0) containing $6.76 \mathrm{mM}$ sodium tartrate, $0.1 \mathrm{mg} / \mathrm{ml}$ naphthol ASMX phosphate and $0.5 \mathrm{mg} / \mathrm{ml}$ Fast Red Violet. The TRAP staining kit was purchased from Sigma-Aldrich; Merck KGaA (Darmstadt, Germany). TRAP-positive multinucleated cells with three or more nuclei were scored and visualized under a microscope (Leica DM 2500; Leica Microsystems GmbH, Wetzlar, Germany).

Hematoxylin and eosin $(H \& E)$ staining. Tibias were fixed with $4 \%$ formalin at room temperature for $24 \mathrm{~h}$, decalcified in $0.5 \mathrm{M}$ EDTA (pH 8.0) and were then embedded in paraffin, according to standard histological procedures. Tibia tissues were cut into $5 \mu \mathrm{m}$ sections, which were stained with H\&E (Beyotime Institute of Biotechnology, Haimen, China), according to the manufacturer's protocol. Stained slides were visualized under an optical microscope (Leica DM 2500; Leica Microsystems $\mathrm{GmbH}$ ) and were analyzed using the OsteoMeasure system (OsteoMetrics Inc., Decatur, GA, USA).

Bone Ca content and BMD. Ca content in the tibia was determined following incineration of the samples using a muffle furnace (Thermo Fisher Scientific, Inc.) at $800^{\circ} \mathrm{C}$ for $12 \mathrm{~h}$. Subsequently, $10 \mathrm{mg}$ bone ash was dissolved in $1 \mathrm{ml} \mathrm{37 \%} \mathrm{HCl}$ diluted with Milli-Q ${ }^{\circledR}$ water. The Ca content was determined using a kit (cat. no. C004-3; Nanjing Jiancheng Bioengineering Institute, Nanjing, China), according to the manufacturer's protocol. The BMD of the tibia was measured by dual-energy X-ray absorptiometry (Lunar DPXIQ; GE Healthcare, Chicago, IL, USA), as described previously (34).

Physiological and biochemical markers in serum and urine samples. The levels of FGF21 in mouse serum were detected using a mouse bioactive ELISA kit (cat. no. E-EL-M0029c; Elabscience Biotechnology, Wuhan, China), according to the manufacturer's protocol. The levels of Ca (cat. no. C004-3), phosphorus (P; cat. no. C006) and creatinine (cat. no. C011-1) in serum and urine samples were measured using kits (Nanjing Jiancheng Bioengineering Institute), according to the manufacturer's protocol. Parathyroid hormone (cat. no. 60-2305; Immutopics, Inc., San Clemente, CA, USA), alkaline phosphatase (cat. no. E-EL-M2720c; ALP; Elabscience Biotechnology), 
A
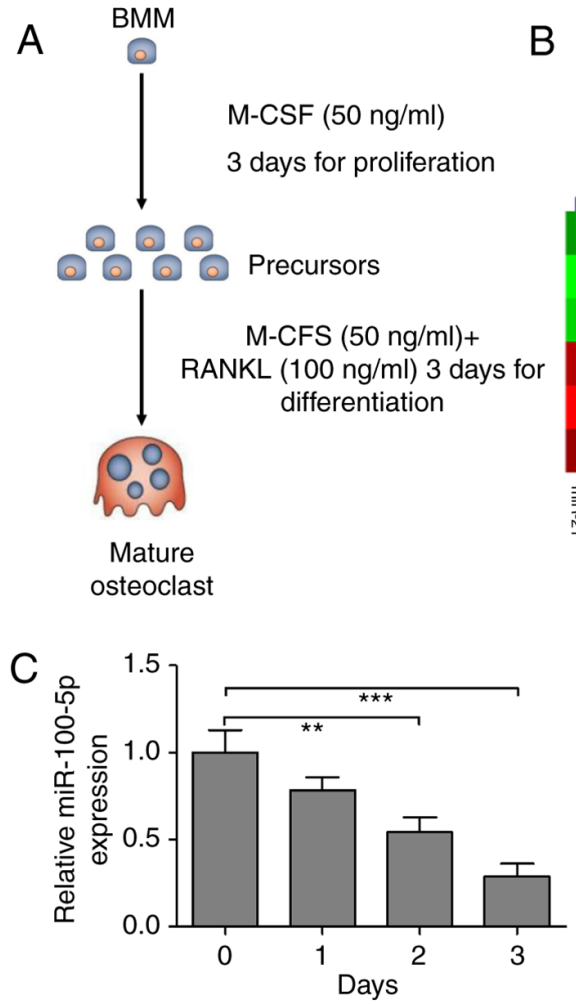

B

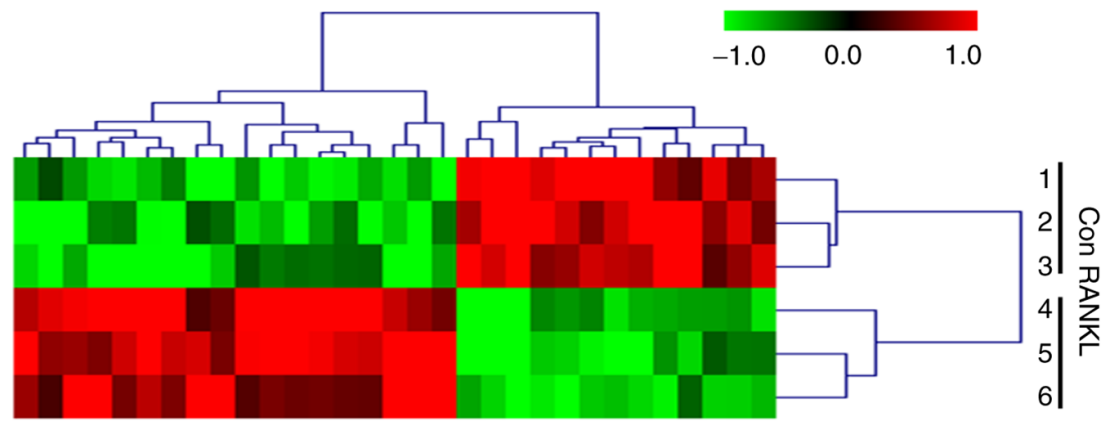

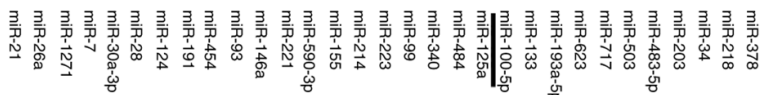

D

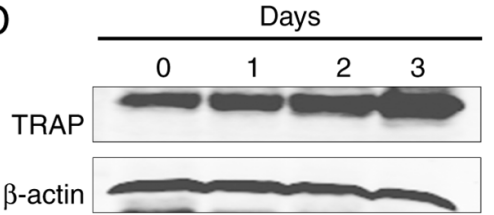

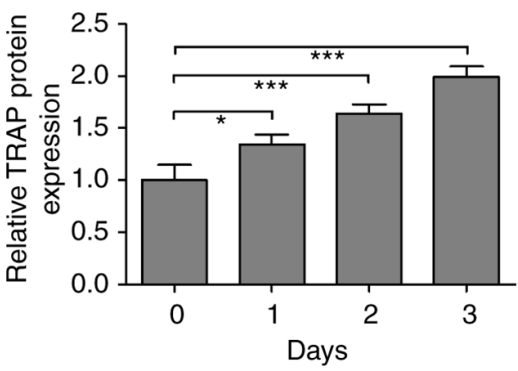

Figure 1. RANKL induces osteoclast differentiation and regulates miRNA expression. (A) Schematic diagram representing the process of M-CSF- and RANKL-induced osteoclastogenesis. (B) Microarray and hierarchical cluster analyses were performed on osteoclast precursors following M-CSF and RANKL stimulation for 3 days. The color legend at the top indicates miRNA expression levels: Red, high expression; green, low expression. (C) Validation of microarray results with regards to miR-100-5p expression by reverse transcription-quantitative polymerase chain reaction analysis. miR-100-5p expression was normalized to U6 expression. (D) Protein expression of TRAP was detected by western blotting in response to M-CSF- and RANKL-induced osteoclastogenesis. Triplicate experiments were performed in each group. ${ }^{*} \mathrm{P}<0.05 ;{ }^{* *} \mathrm{P}<0.01 ;{ }^{* * *} \mathrm{P}<0.001$. M-CSF, macrophage colony-stimulating factor; miRNA/miR, microRNA; RANKL, receptor activator of nuclear factor- $\kappa \mathrm{B}$ ligand; TRAP, tartrate-resistant acid phosphatase.

TRAP-5b (cat. no. SB-TR103; Immunodiagnostic Systems, Inc., Scottsdale, AZ, USA), osteocalcin (cat. no. 60-1305; Immutopics, Inc.) and C-terminal telopeptide of type 1 collagen (CTX; E-EL-M0366c; Elabscience Biotechnology) were detected using mouse bioactive ELISA kits, according to the manufacturers' protocols.

Western blotting. Proteins were extracted from RANKL-stimulated precursor cells, NCTC-1469 cells, and liver and tibia samples using radioimmunoprecipitation assay buffer (Beyotime Institute of Biotechnology). Protein concentration was measured using the Bicinchoninic Acid kit for protein determination (cat. no. BCA1-1KT; Sigma-Aldrich; Merck KGaA). Western blotting was conducted as previously described (35). The membranes were incubated with the following primary antibodies at room temperature for $2 \mathrm{~h}$ : TRAP (cat. no. ab126775; 1:2,000; Abcam, Cambridge, UK), RANK (cat. no. sc-59981; 1:1,000; Santa Cruz Biotechnology, Inc., Dallas, TX, USA), RANKL (cat. no. ab45039; 1:1,000; Abcam), NFATc1 (cat. no. sc-7294; 1:1,000; Santa Cruz Biotechnology, Inc.) and FGF21 (cat. no. ab64857; 1:1,000; Abcam). Following incubation with primary antibodies, the membranes were incubated at room temperature for $1 \mathrm{~h}$ with the appropriate horseradish peroxidase-conjugated anti-mouse secondary antibody (cat. no. sc-516102; 1:10,000; Santa Cruz Biotechnology, Inc.) and anti-rabbit secondary antibody (cat. no. sc-2357; 1:10,000; Santa Cruz Biotechnology, Inc.) and were visualized using chemiluminescence (Thermo Fisher Scientific, Inc.). $\beta$-actin (1:2,000; cat. no. sc-130065; Santa Cruz Biotechnology, Inc.) was used as the control antibody. Signals were analyzed with Quantity One ${ }^{\circledR}$ software version 4.5 (Bio Rad Laboratories, Inc., Hercules, CA, USA).

Luciferase reporter assay. The wild type (WT) or mutant-type (MUT) 3'-UTRs of FGF21 were synthesized by Guangzhou RiboBio Co., Ltd. and were inserted into multiple cloning sites of the luciferase expressing pMIR-REPORT vector (Ambion; Thermo Fisher Scientific, Inc.), according to the manufacturer's protocol. For the luciferase assay, BMMs $\left(1 \times 10^{5}\right)$ were seeded into 24-wells and co-transfected with luciferase reporter vectors containing WT or MUT 3'-UTR $(0.5 \mu \mathrm{g})$ of FGF21, and pre-miR-Con or pre-miR-100-5p (100 nM) using Lipofectamine $^{\circledR} 2000$ (Invitrogen; Thermo Fisher Scientific, Inc.) at $37^{\circ} \mathrm{C}$ for $48 \mathrm{~h}$. Luciferase activity was measured using a dual luciferase reporter assay kit (Beyotime Institute of Biotechnology), according to the manufacturer's protocol; and the WT vector group was used to normalize luciferase activity.

Statistical analysis. Data are presented as the means \pm standard error of the mean. Statistical analysis was performed using SPSS Statistics version 19.0 (IBM Corp., Armonk, NY, USA) and GraphPad Prism version 7.0 (GraphPad Software, Inc., La Jolla, CA, USA). Student's t-test was used to analyze differences between two groups. Inter-group 

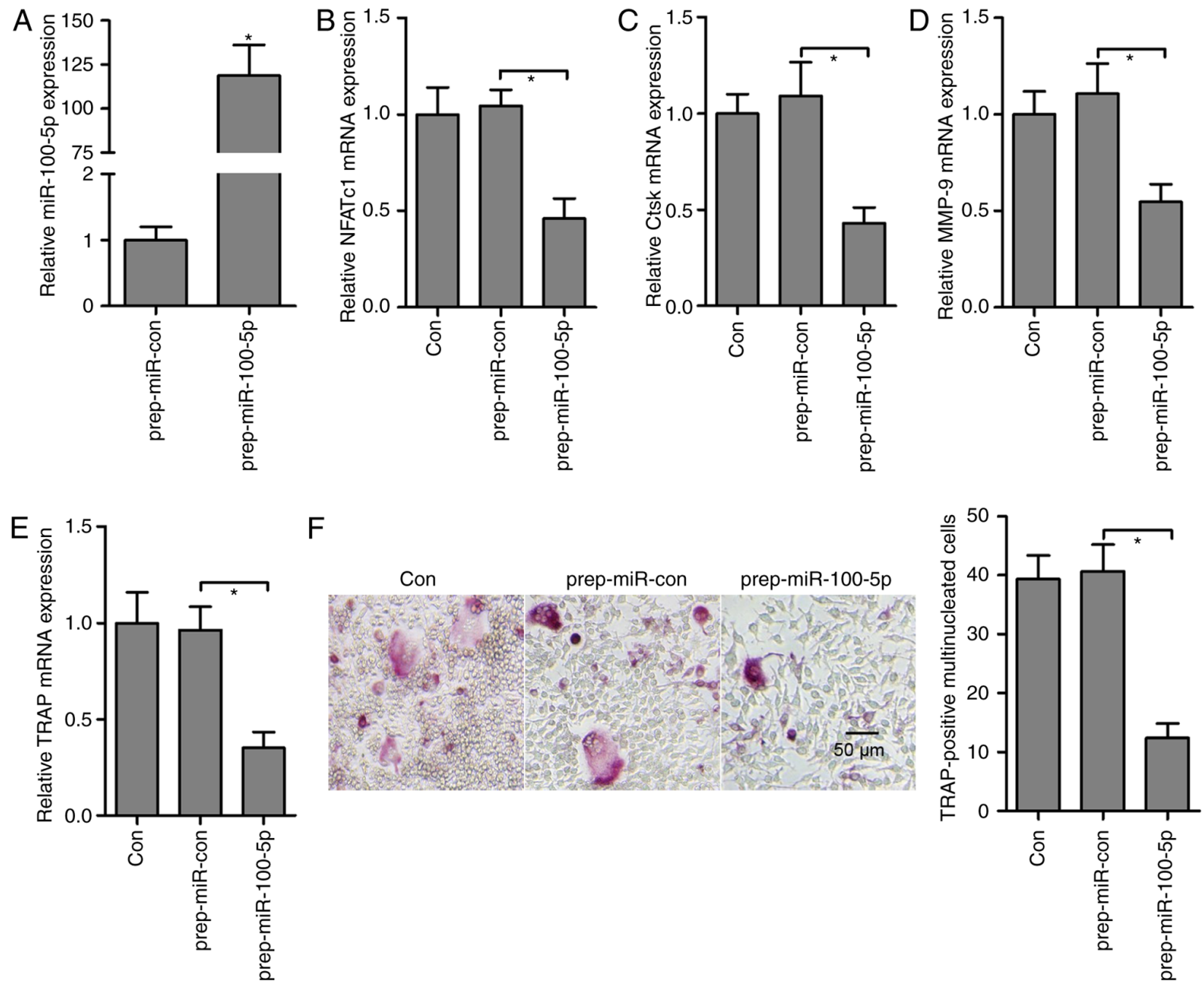

$\mathrm{F}$
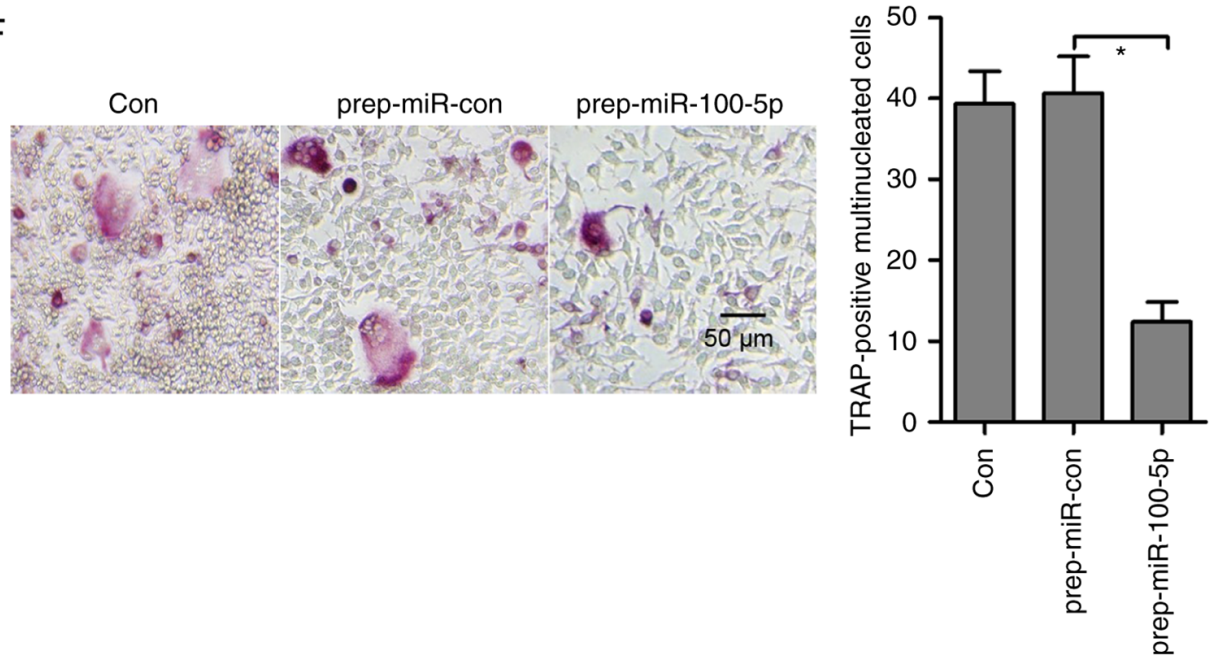

Figure 2. miR-100-5p gain-of-function inhibits osteoclast differentiation. (A) Expression levels of miR-100-5p were measured by RT-qPCR in osteoclast precursors post-transfection with pre-miR-100-5p prior to RANKL stimulation. Expression of osteoclast-specific genes, (B) NFATc1, (C) Ctsk, (D) MMP-9 and (E) TRAP, was detected by RT-qPCR in response to M-CSF- and RANKL-induced osteoclastogenesis and pre-miR-100-5p transfection. (F) Osteoclast precursor cells transfected with pre-miR-100-5p were cultured for an additional 3 days with M-CSF and RANKL, after which, cultured cells were fixed and stained for TRAP, and the number of osteoclasts was counted. Triplicate experiments were performed in each group. " $\mathrm{P}<0.05$. Ctsk, cathepsin K; M-CSF, macrophage colony-stimulating factor; miRNA/miR, microRNA; MMP-9, matrix metalloproteinase-9; NFATc1, nuclear factor of activated T cells, cytoplasmic 1; pre-miR-Con, pre-miR-control; RANKL, receptor activator of nuclear factor- $\mathrm{kB}$ ligand; RT-qPCR, reverse transcription-quantitative polymerase chain reaction; TRAP, tartrate-resistant acid phosphatase.

differences were analyzed by one-way analysis of variance, followed by a post hoc Tukey test for multiple comparisons. $\mathrm{P}<0.05$ was considered to indicate a statistically significant difference.

\section{Results}

miR-100-5p is downregulated during RANKL- and $M-C S F$-induced osteoclastogenesis. BMMs were initially cultured in M-CSF (50 ng/ml) for 3 days to induce formation of osteoclast precursors, which were stimulated by M-CSF $(50 \mathrm{ng} / \mathrm{ml})$ and RANKL $(100 \mathrm{ng} / \mathrm{ml})$ for a further 3 days to induce osteoclastogenesis (Fig. 1A). A miRNA microarray analysis was conducted to determine the molecular mechanism involved in RANKL- and M-CSF-induced osteoclastogenesis. The results demonstrated that 31 miRNAs were differentially expressed in osteoclast precursors with RANKL stimulation, compared with in those without RANKL stimulation, among which 13 and 18 miRNAs were downregulated and upregulated, respectively (Fig. 1B). The expression levels of miR-100-5p were most significantly decreased in RANKL-stimulated cells. In addition, the expression of miR-100-5p was validated by RT-qPCR; the results were consistent with the microarray analysis and indicated that expression was decreased in a time-dependent manner in response to RANKL- and M-CSF-induced osteoclastogenesis (Fig. 1C). Furthermore, the protein expression levels of TRAP were significantly augmented during the process of osteoclastogenesis (Fig. 1D).

Overexpression of miR-100-5p suppresses $R A N K L$ - and $M$-CSF-induced osteoclastogenesis. To investigate the function of miR-100-5p in vitro, pre-miR-100-5p was transfected into osteoclast precursors, in order to amplify the expression of miR-100-5p; the results revealed a significant upregulation ( 120-fold) of miR-100-5p in osteoclast precursors (Fig. 2A). Post-transfection with pre-miR-100-5p, the 
Table II. Biochemical parameters in OVX-operated mice treated with agomir-miR.

\begin{tabular}{lcccc}
\hline Parameters & Sham & OVX & OVX + agomir-con & OVX + agomir-miR \\
\hline Serum Ca (mg/dl) & $10.67 \pm 0.43$ & $9.12 \pm 0.40^{\mathrm{a}}$ & $9.37 \pm 0.32$ & $10.93 \pm 0.45^{\mathrm{b}}$ \\
Serum P $(\mathrm{mg} / \mathrm{dl})$ & $6.06 \pm 0.31$ & $6.12 \pm 0.35$ & $6.25 \pm 0.36$ & $6.17 \pm 0.32$ \\
Urine Ca/Cre $(\mathrm{mg} / \mathrm{mg})$ & $0.114 \pm 0.012$ & $0.223 \pm 0.0^{2} 7^{\mathrm{a}}$ & $0.207 \pm 0.021$ & $0.133 \pm 0.016^{\mathrm{b}}$ \\
Urine P/Cre $(\mathrm{mg} / \mathrm{mg})$ & $1.41 \pm 0.20$ & $1.39 \pm 0.26$ & $1.55 \pm 0.33$ & $1.53 \pm 0.31$ \\
ALP $(\mathrm{ng} / \mathrm{ml})$ & $3.05 \pm 0.23$ & $1.90 \pm 0.21^{\mathrm{a}}$ & $1.75 \pm 0.26$ & $3.82 \pm 0.30$ \\
Osteocalcin $(\mathrm{ng} / \mathrm{ml})$ & $129.3 \pm 15.3$ & $76.7 \pm 16.1^{\mathrm{a}}$ & $68.7 \pm 12.3$ & $110.3 \pm 9.5^{\mathrm{b}}$ \\
TRAP-5b $(\mathrm{U} / \mathrm{l})$ & $8.94 \pm 0.42$ & $12.75 \pm 0.54^{\mathrm{a}}$ & $12.70 \pm 0.58$ & $10.09 \pm 0.45^{\mathrm{b}}$ \\
CTX $(\mathrm{ng} / \mathrm{ml})$ & $15.37 \pm 1.66$ & $27.57 \pm 2.87^{\mathrm{a}}$ & $26.90 \pm 2.22$ & $12.43 \pm 0.83^{\mathrm{b}}$ \\
Serum PTH $(\mathrm{pg} / \mathrm{ml})$ & $123.3 \pm 24.5$ & $256.3 \pm 35.6^{\mathrm{a}}$ & $271.3 \pm 33.2$ & $171.3 \pm 26.6^{\mathrm{b}}$
\end{tabular}

Agomir-Con, control agomir; agomir-miR, miR-100-5p agomir; ALP, alkaline phosphatase; Ca, calcium; Cre, creatinine; CTX, C-terminal telopeptide of type 1 collagen; miR, microRNA; OVX, ovariectomy; P, phosphorus; PTH, parathyroid hormone; TRAP-5b, tartrate-resistant acid phosphatase $5 b$. ${ }^{a} \mathrm{P}<0.05$ vs. the sham-operated group; ${ }^{\text {}} \mathrm{P}<0.05$ vs. the $\mathrm{OVX}+$ Agomir-Con group.

key transcription factor for osteoclastogenesis, NFATc1 (Fig. 2B), and osteoclast-specific genes, Ctsk (Fig. 2C), matrix metalloproteinase-9 (MMP-9) (Fig. 2D) and TRAP (Fig. 2E), were significantly decreased compared with in the pre-miR-Con group. Furthermore, osteoclast differentiation (TRAP-positive multinucleated cells) was blocked by pre-miR-100-5p (Fig. 2F). These findings indicated that miR-100-5p, as a suppressor, may neutralize RANKL- and M-CSF-induced osteoclastogenesis.

Agomir-miR-100-5p blocks OVX-induced bone resorption. The present study also examined whether overexpression of miR-100-5p regulates bone metabolism in vivo. Osteoporotic status was induced by OVX in mice, and OVX-operated mice were administered agomir-miR-100-5p or agomir-Con via a tail vein injection. ELISA analyses revealed that bone formation markers, ALP and osteocalcin, and bone resorption markers, TRAP-5b and CTX, were downregulated and upregulated, respectively, in OVX-operated mice. Conversely, agomir-miR-100-5p treatment reversed OVX-induced marker alterations, with the exception of serum ALP (Table II).

RT-qPCR analysis revealed that miR-100-5p levels were significantly downregulated in the tibia from OVX-operated mice compared with in the tibia from sham-operated mice, whereas in OVX-operated mice injected with agomir-miR-100-5p OVX-inhibited miR-100-5p levels were markedly rescued in the tibia (Fig. 3A). Subsequently, the mRNA expression levels of osteoblast-specific genes [Runt-related transcription factor 2 (Runx2), osterix, osteocalcin and ALP] were detected, and it was confirmed that these genes were reduced in the tibia from OVX-operated mice, whereas agomir-miR-100-5p administration in OVX-operated mice significantly upregulated the mRNA expression levels of Runx2, osterix, osteocalcin and ALP (Fig. 3B). Conversely, the mRNA ratio of RANKL/osteoprotegrin (OPG) was elevated in the tibia from OVX-operated mice, suggesting that an increase in osteoclast activity in OVX-operated mice may be associated with overactivation of RANKL availability; however, upregulation of the RANKL/OPG ratio was reversed by agomir-miR-100-5p administration in OVX-operated mice (Fig. 3C). To further investigate whether miR-100-5p inhibited OVX-induced bone resorption, osteoclast-specific genes, NFATc1, Ctsk, MMP-9, carbonic anhydrase 2 (CA2) and TRAP, were detected by RT-qPCR; the results demonstrated that agomir-miR-100-5p treatment markedly abolished OVX-induced upregulation of osteoclast-specific genes in the tibia (Fig. 3D). In addition, as determined by western blotting, OVX-operated mice exhibited a significant increase in RANK, RANKL and NFATc1, whereas agomir-miR-100-5p administration decreased the protein expression levels of RANK, RANKL and NFATc1 in the tibia from OVX-operated mice(Fig. 3E and 3F).

$\mathrm{Ca}$ and $\mathrm{P}$ metabolism were also analyzed in OVX-operated mice in response to agomir-miR-100-5p administration. Compared with in agomir-Con treated OVX-operated mice, agomir-miR-100-5p administration significantly increased serum $\mathrm{Ca}$ concentration and blocked urinary $\mathrm{Ca}$ secretion; however, the serum and urine levels of $\mathrm{P}$ were not markedly altered in response to OVX operation or agomir-miR-100-5p administration (Table II). Bone Ca content and tibia BMD were decreased by OVX and were increased by agomir-miR-100-5p administration in OVX-operated mice (Fig. 4A and B). Histomorphological observation by H\&E staining revealed that the integrity of the trabecular bone microstructure network was destroyed in the proximal epiphysis of the tibia by OVX. However, agomir-miR-100-5p administration markedly improved trabecular bone structure and accelerated bone remodeling (Fig. 4C and D). Furthermore, the function of agomir-miR-100-5p on osteoclast differentiation was determined in vivo. TRAP staining revealed that the number of osteoclasts was increased by OVX, whereas osteoclast numbers were significantly decreased by agomir-miR-100-5p treatment (Fig. 4E and F). Collectively, these findings suggested that increased bone mass in agomir-miR-100-5p-administered OVX-operated mice may be caused by a combination of increased bone formation and decreased bone resorption.

Agomir-miR-100-5p abolishes OVX-induced FGF21 in the liver. Previous studies have reported that FGF21 is 
A

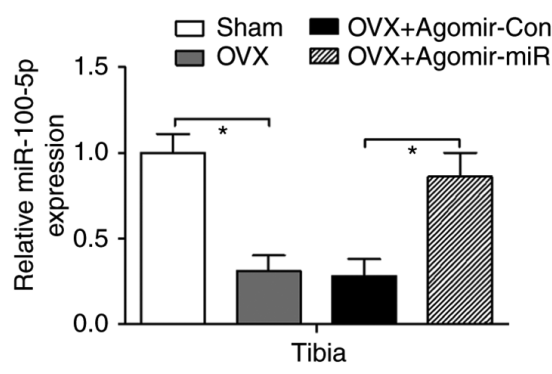

B

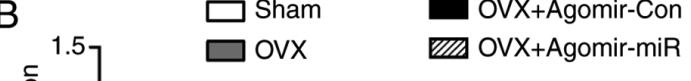

C

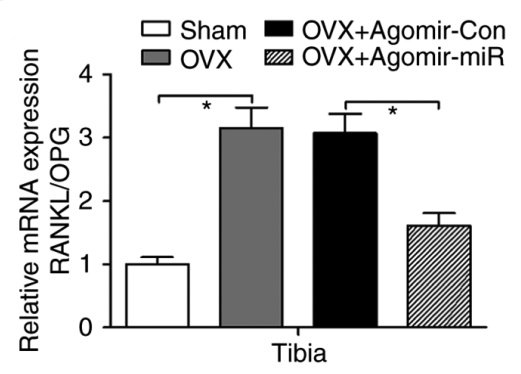

$\mathrm{D}$

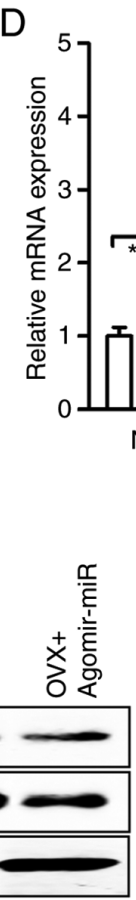

$E$

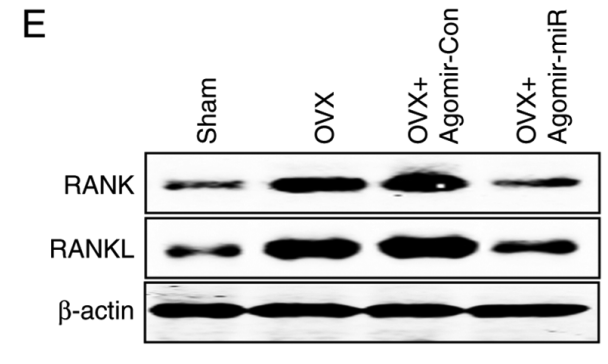

$\mathrm{F}$

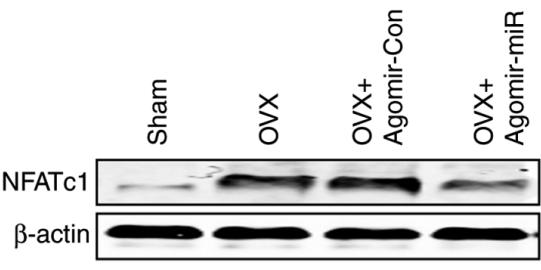

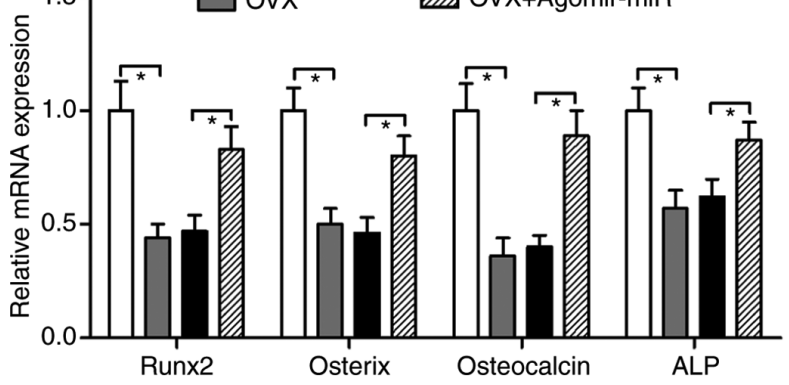

OVX+Agomir-Con

m OVX+Agomir-miR

$\square$ ovx
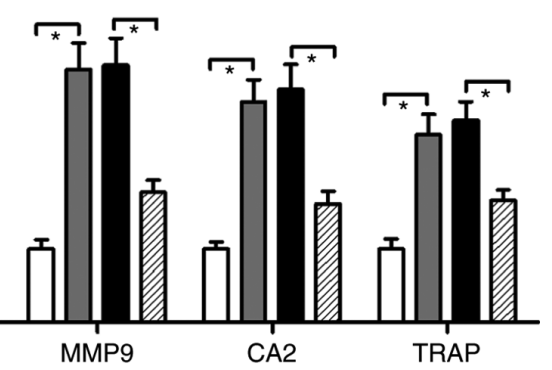
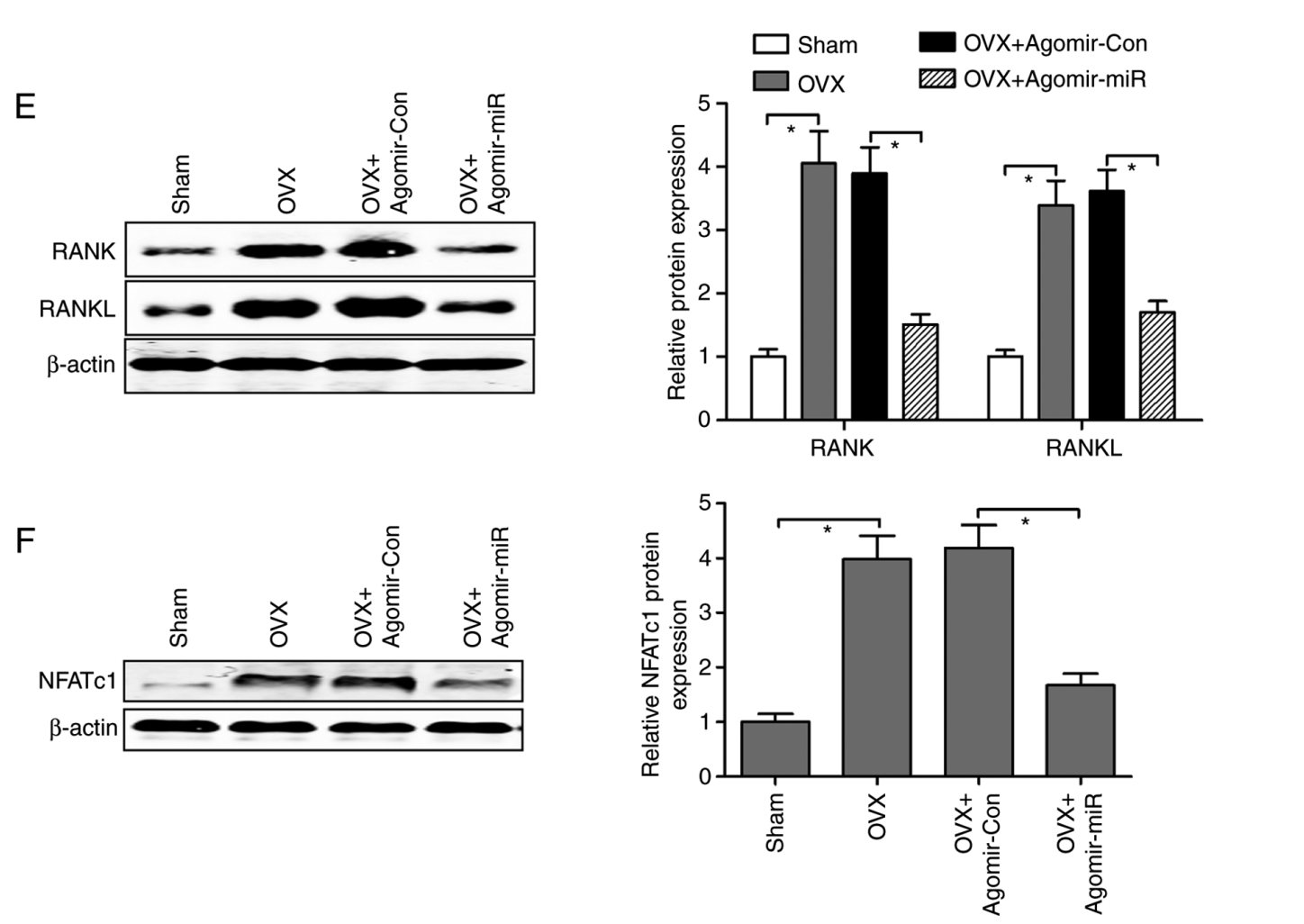

Figure 3. Agomir-miR regulates osteoblast- and osteoclast-specific gene expression in OVX-operated mice. (A) Mice in the OVX group were injected intravenously with agomir-miR-100-5p or agomir-Con, and the expression levels of miR-100-5p were measured by RT-qPCR in the tibia. (B) Osteoblast-specific genes, Runx2, osterix, osteocalcin and ALP, were detected in the tibia by RT-qPCR. (C) Ratio of RANKL mRNA to OPG mRNA in the tibia was calculated as a marker of osteoclast activity. (D) RT-qPCR was performed to analyze the mRNA expression levels of osteoclast-specific genes in the tibia. (E and F) Protein expression levels of RANK, RANKL and NFATc1 in the tibia were detected using western blotting. Triplicate experiments were performed in each group. "P<0.05. agomir-miR, miR-100-5p agomir; agomir-Con, control agomir; CA2, carbonic anhydrase 2; Ctsk, cathepsin K; miR, microRNA; MMP-9, matrix metalloproteinase-9; NFATc1, nuclear factor of activated T cells, cytoplasmic 1; OPG, osteoprotegrin; OVX, ovariectomy; RANK, receptor activator of nuclear factor- $\mathrm{B}$ RANKL, RANK ligand; RT-qPCR, reverse transcription-quantitative polymerase chain reaction; TRAP, tartrate-resistant acid phosphatase.

inducible in the liver, but absent in the bone, and indirectly promotes osteoclastogenesis and bone resorption via simultaneously decreasing bone formation and increasing bone resorption $(26,30)$. To determine the association between miR-100-5p and FGF21, agomir-miR-100-5p or agomir-Con were injected into OVX-operated mice via the tail vein; the 
A

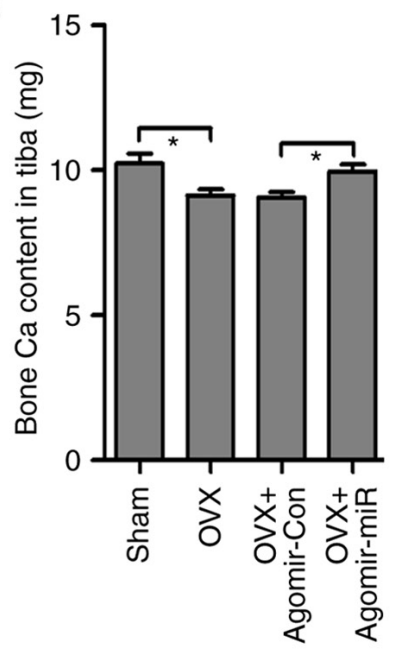

B

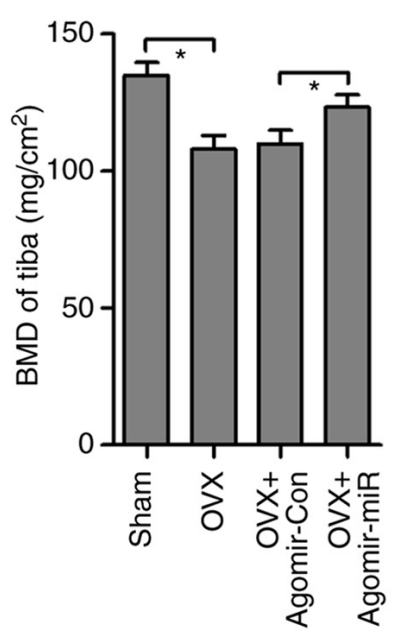

C

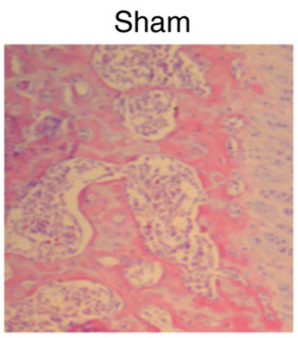

OVX+Agomir-Con

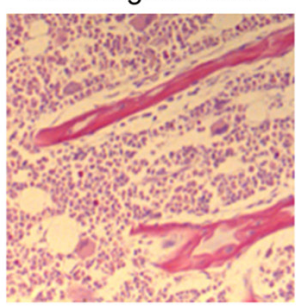

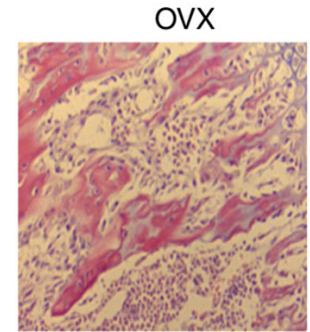

OVX+Agomir-miR

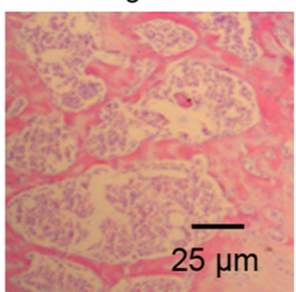

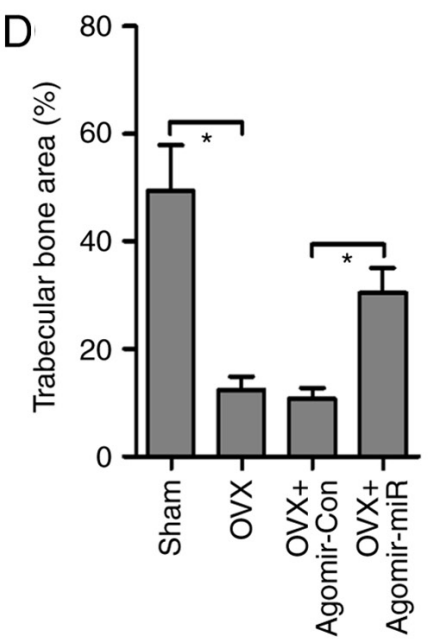
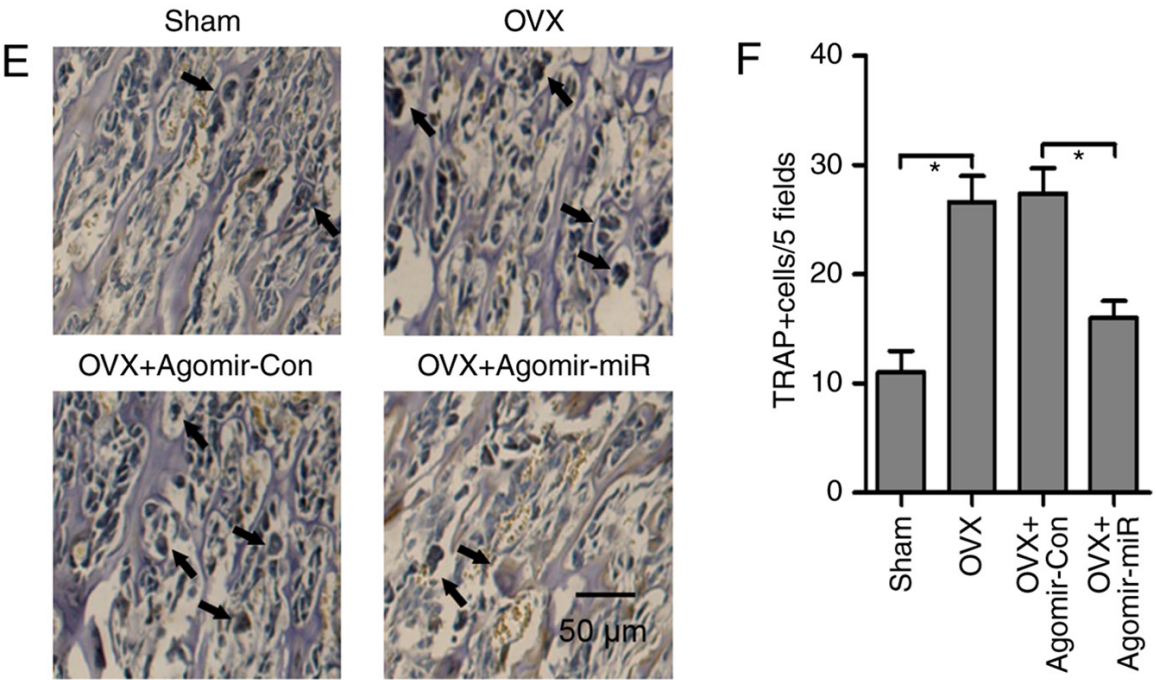

Figure 4. Agomir-miR inhibits bone loss in the tibia from OVX-operated mice. (A) Bone Ca content and (B) tibia BMD were measured in OVX-operated mice treated with agomir-miR or agomir-Con injection. (C) Hematoxylin and eosin staining was performed to assess the effects of agomir-miR on trabecular bone microstructure in the proximal tibia from OVX-operated mice (magnification, x200; scale bar, $25 \mu \mathrm{m}$ ). (D) Ratio of trabecular bone area to total area in the proximal tibia was calculated to estimate trabecular bone metabolism. (E) TRAP staining (magnification, x100; scale bar, $50 \mu \mathrm{m}$ ) and (F) number of osteoclasts in the tibia were measured to assess the effects of agomir-miR on osteoclast activity (black arrows represent mature osteoclasts). Triplicate experiments were performed in each group. " $\mathrm{P}<0.05$. Agomir-Con, control agomir; agomir-miR, miR-100-5p agomir; BMD, bone mineral density; Ca, calcium; miR, microRNA; OVX, ovariectomy; TRAP, tartrate-resistant acid phosphatase.

results revealed that miR-100-5p was significantly decreased in the liver of OVX-operated mice compared with in the sham-operated group, whereas agomir-miR-100-5p injection rescued OVX-induced downregulation of miR-100-5p expression in the liver (Fig. 5A). Consistent with previous findings $(26,30)$, the present results indicated a significant increase in the mRNA expression (Fig. 5B), serum levels (Fig. 5C) and protein expression (Fig. 5D and E) of FGF21 in the liver of OVX-operated mice. Notably, both liver and serum FGF21 levels were suppressed by agomir-miR-100-5p injection in OVX-operated mice (Fig. 5B-E).

To further investigate whether FGF21 is a direct target of miR-100-5p, the online prediction software TargetScan (http://www.targetscan.org/vert_72/) was used to predict the association between miR-100-5p and FGF21. It was revealed that the 3'-UTR of FGF21 contained one conserved binding site for miR-100-5p, which was presented as a complementary pairing (Fig. 6A). To confirm this finding, luciferase reporter plasmids containing WT or MUT 3'-UTR of FGF21 were co-transfected with pre-miR-Con and pre-miR-100-5p into the NCTC-1469 mouse normal liver cell line. The results revealed that the luciferase activity was reduced by $\sim 60 \%$ in cells transfected with plasmids containing WT 3'-UTR of FGF21 and pre-miR-100-5p; however, pre-miR-100-5p had no effect on luciferase activity in cells transfected with plasmids containing MUT 3'-UTR of FGF21 (Fig. 6B). Furthermore, to identify the action of miR-100-5p on FGF21, NCTC-1469 cells were transfected with pre-miR-Con or pre-miR-100-5p and the mRNA and protein expression levels of FGF21 were measured. Compared with in the pre-miR-Con group, overexpression of miR-100-5p significantly downregulated the mRNA and protein expression levels of FGF21 (Fig. 6C and D). These findings suggested that FGF21 may be a direct target of miR-100-5p. 
A

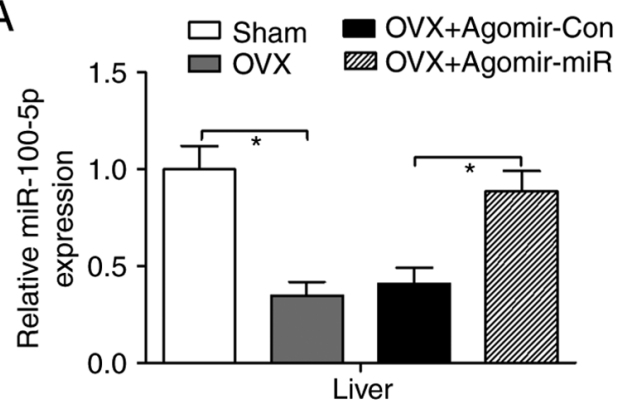

B

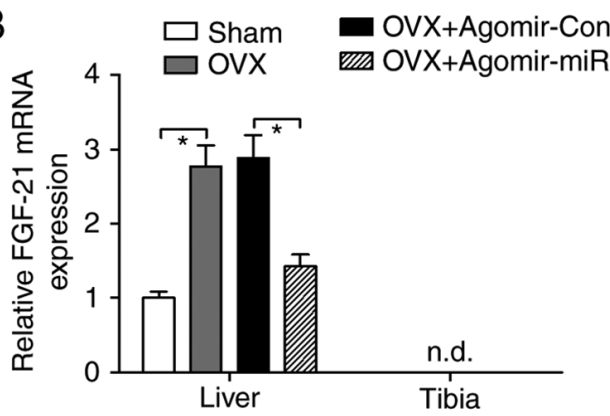

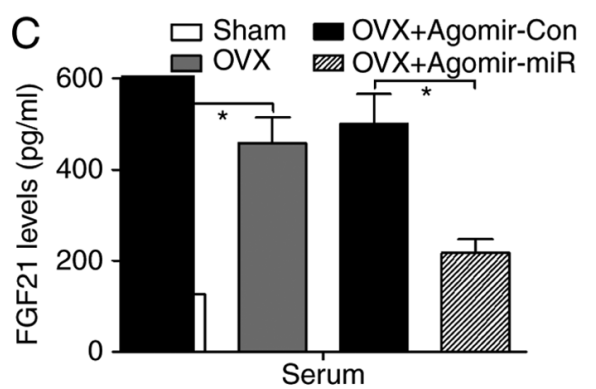
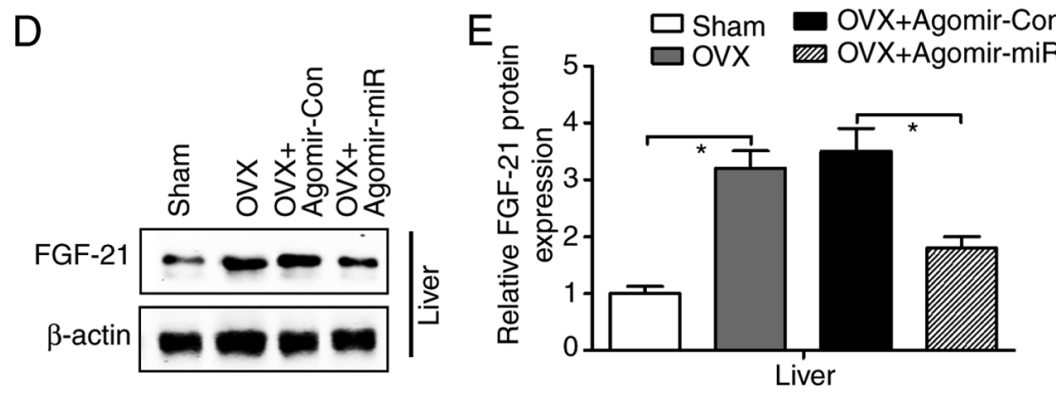

Figure 5. Mice in the OVX group were injected intravenously with agomir-miR or agomir-Con. (A) miR-100-5p expression in the liver was measured by RT-qPCR; (B) mRNA expression of FGF21 in the liver and tibia was measured by RT-qPCR; (C) serum levels of FGF21 were measured by ELISA; (D and E) protein expression of TRAP in the liver was detected by western blotting. Triplicate experiments were performed in each group. ${ }^{*} \mathrm{P}<0.05$. Agomir-Con, control agomir; agomir-miR, miR-100-5p agomir; FGF21, fibroblast growth factor 21; n.d., not detected; OVX, ovariectomy; TRT-qPCR, reverse transcription-quantitative polymerase chain reaction; TRAP, tartrate-resistant acid phosphatase.

A
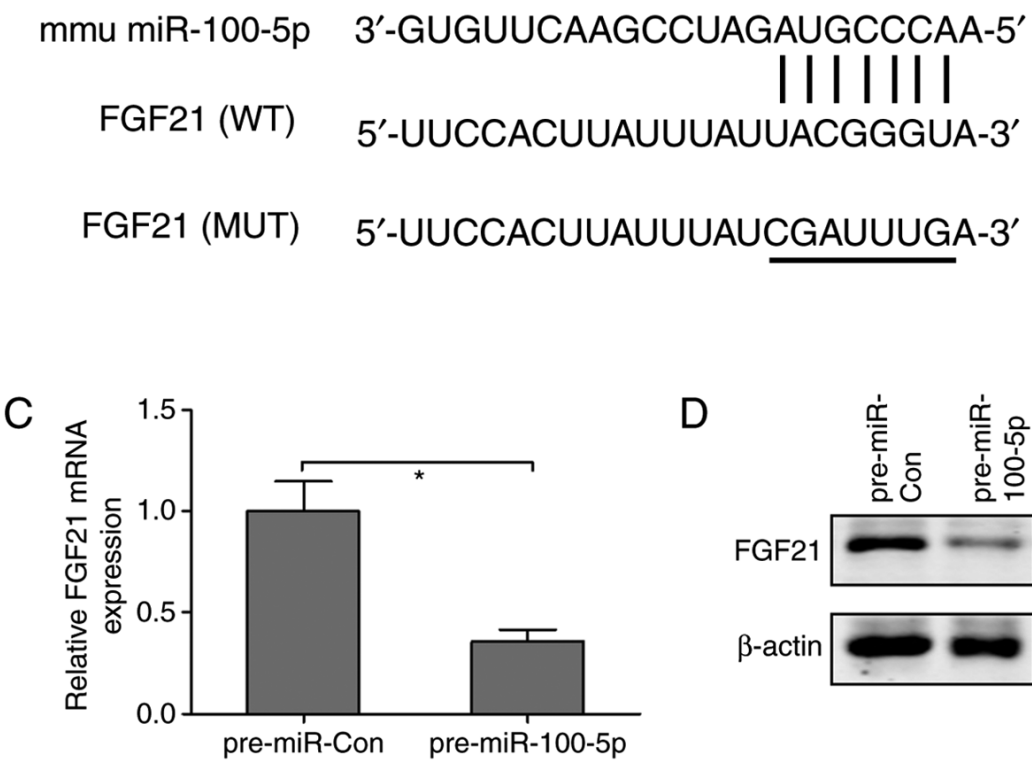

B
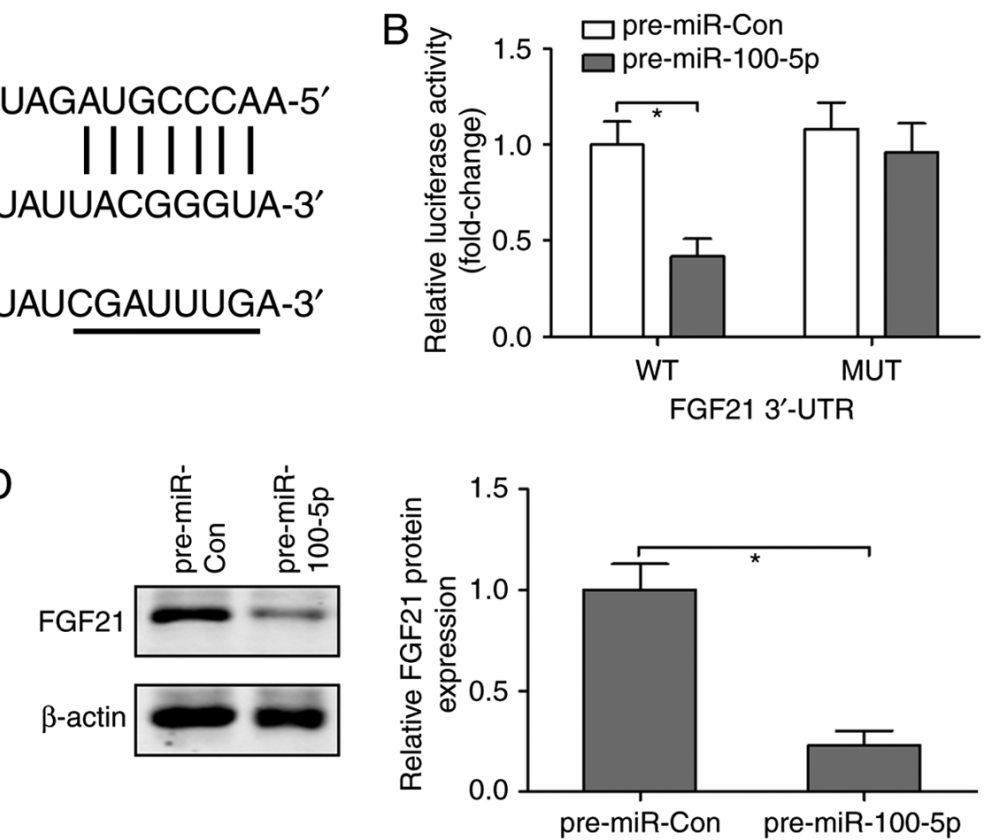

Figure 6. FGF21 is a direct target of miR-100-5p. (A) Putative miR-100-5p binding sites in the $3^{\prime}-$ UTR of FGF21 were predicted by bioinformatics analysis. (B) Luciferase activity assay was performed post-transfection with pre-miR-100-5p or pre-miR-Con in NCTC-1469 cell with WT or MUT 3'-UTR of FGF21. (C) mRNA and (D) protein expression levels of FGF21 were assessed by reverse transcription-quantitative polymerase chain reaction and western blotting, respectively, post-transfection with pre-miR-100-5p or pre-miR-Con in NCTC-1469 cells. Triplicate experiments were performed in each group. " $\mathrm{P}<0.05$. 3'-UTR, 3'-untranslated region; Con, control; FGF21, fibroblast growth factor 21; miR, microRNA; MUT, mutant-type; pre-miR-Con, pre-miR-control; WT, wild type.

Based on the aforementioned findings, it was revealed that miR-100-5p was inhibited during the process of osteoclastogenesis in vitro and in OVX-induced bone loss in vivo. FGF21, as a potential pro-osteoclastogenic liver hormone, may be involved in RANKL/RANK/NFATc1 cascade signaling-mediated osteoclast differentiation, whereas agomir-miR-100-5p, as a post-transcriptional regulatory factor targeted to the FGF21/RANKL/RANK/NFATc1 pathway, may block osteoclastogenesis and OVX-induced bone loss (Fig. 7). 


\section{Discussion}

The present study confirmed that the expression of miR-100-5p was downregulated during RANKL-induced osteoclastogenesis, as determined by microarray and RT-qPCR analyses. miR-100-5p gain-of-function markedly inhibited the process of osteoclast differentiation and negatively regulated osteoclast function by suppressing the mRNA expression levels of osteoclast-specific genes in vitro. The present results also suggested that $\mathrm{Ca}$ metabolism and trabecular bone remodeling were improved, and osteoclast differentiation and activity were decreased by agomir-miR-100-5p in OVX-operated mice. Furthermore, as a crucial liver hormone, FGF21 was upregulated and involved in OVX-induced bone resorption. Notably, agomir-miR-100-5p abrogated OVX-induced upregulation of FGF21 in the liver and serum; FGF21 was validated as a direct target of miR-100-5p by bioinformatics analysis and luciferase activity assay. Overexpression of miR-100-5p resulted in the downregulation of FGF21 mRNA and protein expression in NCTC-1469 cells. Based on these findings, it was suggested that agomir-miR-100-5p alleviated osteoclast differentiation and bone loss by blocking liver-secreted FGF-21; FGF-21 may lead to overactivation of the RANKL/RANK/NFATc1 signaling pathway, thus mediating osteoclastic bone resorption.

miRNAs are post-transcriptional regulators that mediate the expression of bone metabolism-associated genes at transcriptional and translational levels $(9-11,13)$. For example, overexpression of miR-503 prevents bone resorption and osteoclast activity in vitro and in vivo by inhibiting RANK expression (11). miR-34a, as a key osteoclast suppressor, blocks osteoporosis by suppressing transforming growth factor- $\beta$-induced factor 2 in mice (9). Furthermore, miR-214 leads to a decline in bone formation by inhibiting activating transcription factor 4 and phosphatase and tensin homolog $(10,13)$. These results suggested that a regulatory mechanism including numerous miRNAs, or one miRNA, may target various genes involved in the process of osteoclastogenesis and bone loss $(9-11,13)$. A previous study reported that miR-100 modulates human mesenchymal stem cell (MSC) differentiation into osteoblasts, and promotes osteogenesis in response to mechanical stress (36). Conversely, miR-100 serves a negative role in osteogenic differentiation derived from human adipose-derived MSCs by targeting bone morphogenetic protein receptor type II (18). The present study revealed that downregulation of miR-100-5p was accompanied by an increase in the expression levels of FGF21 in the liver samples of OVX-operated mice. Further investigations indicated that FGF21 was a direct target of miR-100-5p, which could inhibit FGF21 levels in the serum and liver; osteoclast activity and bone loss were suppressed by agomir-miR-100-5p injection in OVX-operated mice. These observations provided an insight into liver-bone endocrine signaling and suggested that miR-100-5p targeted FGF21 to inhibit its hepatic expression and contributed to the regeneration of bone under pathophysiological conditions.

FGF21 is a hepatokine that is mainly expressed in the liver $(26,30)$. FGF21 is considered a potential novel drug for the treatment of obesity and diabetes by potentiating the effect

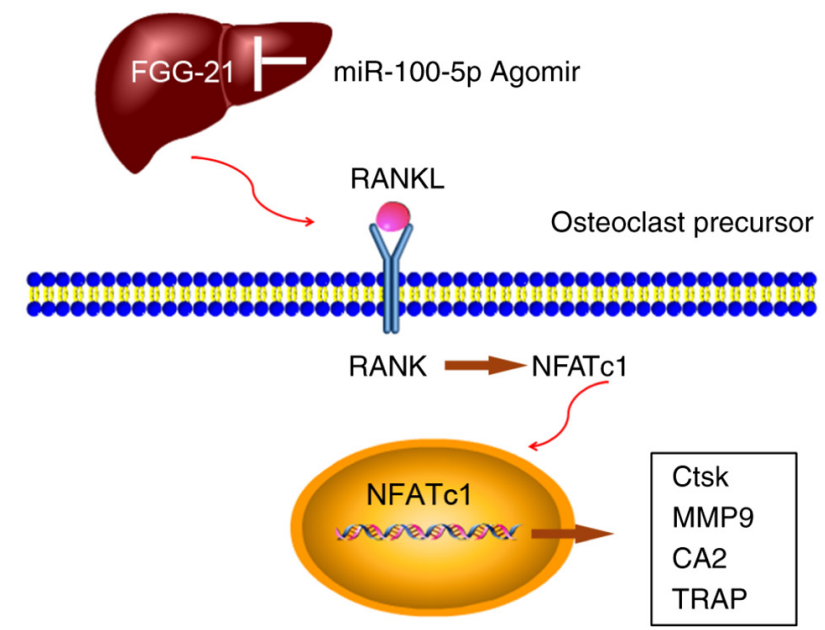

Osteoclast differentiation

Figure 7. Schematic diagram of how miR-100-5p may mediate the suppression of osteoclastogenesis by targeting the hepatokine FGF-21. CA2, carbonic anhydrase 2; Ctsk, cathepsin K; FGF21, fibroblast growth factor 21; miR, microRNA; MMP-9, matrix metalloproteinase-9; NFATc1, nuclear factor of activated T cells, cytoplasmic 1; RANK, receptor activator of nuclear factor- $\kappa \mathrm{B}$; RANKL, RANK ligand.

of peroxisome proliferator-activated receptor $\gamma($ PPAR- $\gamma)$ (37). Furthermore, FGF21 inhibits osteoblastogenesis from MSCs and stimulates osteoclastogenesis from hematopoietic stem cells by activating PPAR- $\gamma$, whereas bone mass is markedly restored by FGF21 deletion (26,37). A previous study (36), and the present findings, revealed that miR-100-5p exerted an opposite effect to FGF21 on osteoclast differentiation. A functional study revealed that FGF21 was a direct target of miR-100-5p, and agomir-miR-100-5p effectively blocked FGF21 secretion from the liver, inhibited osteoclastogenesis and osteoclast-specific gene expression, and stimulated bone formation and osteoblast-specific gene expression. FGF21 was inducible in the liver but absent in the bone, thus suggesting that FGF21-stimulated osteoclast differentiation and bone loss may be mediated via a liver-bone endocrine metabolic mechanism in OVX-operated mice.

In conclusion, the present results demonstrated that alterations in the expression levels of miR-100-5p may lead to an alteration in bone homeostasis and osteoclast differentiation. These phenomena may be associated with the hepatokine FGF-21. The present experimental evidence from in vitro and in vivo studies strongly indicated that miR-100-5p could prevent bone resorption and osteoclastogenesis, at least partially, through inhibition of FGF-21 secretion from the liver. This finding may provide a novel insight into the management of osteoporosis and osteoclast-associated bone diseases.

\section{Acknowledgements}

Not applicable.

\section{Funding}

The present study was supported by the National Natural Science Foundation of China (grant no. 81370981). 


\section{Availability of data and materials}

All data generated or analyzed during this study are included in this published article.

\section{Authors' contributions}

The study was designed by LZ, HYS, LLG, LYY, SM and QF. Literature research, data acquisition and data analysis were performed by LZ, HYS and QF. Histological examination was conducted by LLG, LYY and SM. Western blotting and RT-qPCR were performed by LZ, HYS and QF. The manuscript was prepared and edited by LZ, HYS, LLG, LYY, SM and QF. The manuscript was reviewed by LZ, HYS, LLG, LYY, SM and QF. QF approved the final version of the manuscript.

\section{Ethics approval and consent to participate}

The present study was approved by the Animal Ethical Committee of Shengjing Hospital of China Medical University (approval no. 2016PS361K).

\section{Patient consent for publication}

Not applicable.

\section{Competing interests}

The authors declare that they have no competing interests.

\section{References}

1. Yuan FL, Wu QY, Miao ZN, Xu MH, Xu RS, Jiang DL, Ye JX, Chen FH, Zhao MD, Wang HJ and Li X: Osteoclast-derived extracellular vesicles: Novel regulators of osteoclastogenesis and osteoclast-osteoblasts communication in bone remodeling. Front Physiol 9: 628, 2018.

2. Neman J, Hambrecht A, Cadry C and Jandial R: Stem cell-mediated osteogenesis: Therapeutic potential for bone tissue engineering. Biologics 6: 47-57, 2012.

3. Xiao W, Wang Y, Pacios S, Li S and Graves DT: Cellular and molecular aspects of bone remodeling. Front Oral Biol 18: 9-16, 2016.

4. Deng L, Wang Y, Peng Y, Wu Y, Ding Y, Jiang Y, Shen Z and Fu Q: Osteoblast-derived microvesicles: A novel mechanism for communication between osteoblasts and osteoclasts. Bone 79: $37-42,2015$.

5. Siddiqi MH, Siddiqi MZ, Ahn S, Kang S, Kim YJ, Sathishkumar N, Yang DU and Yang DC: Ginseng saponins and the treatment of osteoporosis: Mini literature review. J Ginseng Res 37: 261-268, 2013.

6. Collison J: Bone: miR-106b promotes osteoporosis in mice. Nat Rev Rheumatol 13: 130, 2017.

7. Szabo G and Bala S: MicroRNAs in liver disease. Nat Rev Gastroenterol Hepatol 10: 542-552, 2013.

8. Rottiers V and Naar AM: MicroRNAs in metabolism and metabolic disorders. Nat Rev Mol Cell Biol 13: 239-250, 2012.

9. Krzeszinski JY, Wei W, Huynh H, Jin Z, Wang X, Chang TC, Xie XJ, He L, Mangala LS, Lopez-Berestein G, et al: miR-34a blocks osteoporosis and bone metastasis by inhibiting osteoclastogenesis and Tgif2. Nature 512: 431-435, 2014.

10. Wang X, Guo B, Li Q, Peng J, Yang Z, Wang A, Li D, Hou Z, Lv K, Kan G, et al: miR-214 targets ATF4 to inhibit bone formation. Nat Med 19: 93-100, 2013.

11. Chen C, Cheng P, Xie H, Zhou HD, Wu XP, Liao EY and Luo XH: MiR-503 regulates osteoclastogenesis via targeting RANK. J Bone Miner Res 29: 338-347, 2014.
12. Guo LJ, Liao L, Yang L, Li Y and Jiang TJ: MiR-125a TNF receptor-associated factor 6 to inhibit osteoclastogenesis. Exp Cell Res 321: 142-152, 2014.

13. Zhao C, Sun W, Zhang P, Ling S, Li Y, Zhao D, Peng J, Wang A, Li Q, Song J, et al: miR-214 promotes osteoclastogenesis by targeting Pten/PI3k/Akt pathway. RNA Biol 12: 343-353, 2015.

14. Jin Y, Tymen SD, Chen D, Fang ZJ, Zhao Y, Dragas D, Dai Y, Marucha PT and Zhou X: MicroRNA-99 family targets AKT/mTOR signaling pathway in dermal wound healing. PLoS One 8: e64434, 2013.

15. Lu Y, Zhao X, Liu Q, Li C, Graves-Deal R, Cao Z, Singh B, Franklin JL, Wang J, Hu H, et al: IncRNA MIR100HG-derived miR-100 and miR-125b mediate cetuximab resistance via Wnt/ $\beta$-catenin signaling. Nat Med 23: 1331-1341, 2017.

16. Liu M, Han T, Shi S and Chen E: Long noncoding RNA HAGLROS regulates cell apoptosis and autophagy in lipopolysaccharides-induced WI-38 cells via modulating miR-100/NF- $\kappa$ B axis. Biochem Biophys Res Commun 500: 589-596, 2018.

17. Seeliger C, Karpinski K, Haug AT, Vester H, Schmitt A, Bauer JS and van Griensven M: Five freely circulating miRNAs and bone tissue miRNAs are associated with osteoporotic fractures. J Bone Miner Res 29: 1718-1728, 2014.

18. Zeng Y, Qu X, Li H, Huang S, Wang S, Xu Q, Lin R, Han Q, Li J and Zhao RC: MicroRNA-100 regulates osteogenic differentiation of human adipose-derived mesenchymal stem cells by targeting BMPR2. FEBS Lett 586: 2375-2381, 2012.

19. Liu J, $\mathrm{Xu} \mathrm{Y,} \mathrm{Hu} \mathrm{Y} \mathrm{and} \mathrm{Wang} \mathrm{G:} \mathrm{The} \mathrm{role} \mathrm{of} \mathrm{fibroblast} \mathrm{growth}$ factor 21 in the pathogenesis of non-alcoholic fatty liver disease and implications for therapy. Metabolism 64: 380-390, 2015.

20. Charoenphandhu N, Suntornsaratoon P, Krishnamra N, Sa-Nguanmoo P, Tanajak P, Wang X, Liang G, Li X, Jiang C, Chattipakorn $\mathrm{N}$ and Chattipakorn S: Fibroblast growth factor-21 restores insulin sensitivity but induces aberrant bone microstructure in obese insulin-resistant rats. J Bone Miner Metab 35: 142-149, 2017.

21. Anuwatmatee S, Tang S, Wu BJ, Rye KA and Ong KL: Fibroblast growth factor 21 in chronic kidney disease. Clin Chim Acta S0009-8981: 30432-1, 2017.

22. Planavila A, Redondo I, Hondares E, Vinciguerra M, Munts C, Iglesias R, Gabrielli LA, Sitges M, Giralt M, van Bilsen M and Villarroya F: Fibroblast growth factor 21 protects against cardiac hypertrophy in mice. Nat Commun 4: 2019, 2013.

23. Desai BN, Singhal G, Watanabe M, Stevanovic D, Lundasen T, Fisher FM, Mather ML, Vardeh HG, Douris N, Adams AC, et al: Fibroblast growth factor 21 (FGF21) is robustly induced by ethanol and has a protective role in ethanol associated liver injury. Mol Metab 6: 1395-1406, 2017.

24. Li H, Dong K, Fang Q, Hou X, Zhou M, Bao Y, Xiang K, Xu A and Jia W: High serum level of fibroblast growth factor 21 is an independent predictor of non-alcoholic fatty liver disease: A 3-year prospective study in China. J Hepatol 58: 557-563, 2013.

25. Samson SL, Sathyanarayana P, Jogi M, Gonzalez EV, Gutierrez A, Krishnamurthy R, Muthupillai R, Chan L and Bajaj M: Exenatide decreases hepatic fibroblast growth factor 21 resistance in non-alcoholic fatty liver disease in a mouse model of obesity and in a randomised controlled trial. Diabetologia 54: 3093-3100, 2011.

26. Wei W, Dutchak PA, Wang X, Ding X, Wang X, Bookout AL, Goetz R, Mohammadi M, Gerard RD, Dechow PC, et al: Fibroblast growth factor 21 promotes bone loss by potentiating the effects of peroxisome proliferator-activated receptor $\gamma$. Proc Natl Acad Sci USA 109: 3143-3148, 2012.

27. Hao RH, Gao JL, Li M, Huang W, Zhu DL, Thynn HN, Dong SS and Guo Y: Association between fibroblast growth factor 21 and bone mineral density in adults. Endocrine 59: 296-303, 2018.

28. Gallego-Escuredo JM, Lamarca MK, Villarroya J, Domingo JC, Mateo MG, Gutierrez MDM, Vidal F, Villarroya F, Domingo P and Giralt M: High FGF21 levels are associated with altered bone homeostasis in HIV-1-infected patients. Metabolism 71: 163-170, 2017.

29. Lee P, Linderman J, Smith S, Brychta RJ, Perron R, Idelson C, Werner CD, Chen KY and Celi FS: Fibroblast growth factor 21 (FGF21) and bone: Is there a relationship in humans? Osteoporos Int 24: 3053-3057, 2013.

30. Wang X, Wei W, Krzeszinski JY, Wang Y and Wan Y: A liver-bone endocrine relay by IGFBP1 promotes osteoclastogenesis and mediates FGF21-induced bone resorption. Cell Metab 22: 811-824, 2015. 
31. Zhao H, Zhang J, Shao H, Liu J, Jin M, Chen J and Huang Y: Transforming growth factor $\beta 1 / \mathrm{Smad} 4$ signaling affects osteoclast differentiation via regulation of miR-155 expression. Mol Cells 40: 211-221, 2017.

32. Saeed AI, Sharov V, White J, Li J, Liang W, Bhagabati N, Braisted J, Klapa M, Currier T, Thiagarajan M, et al: TM4: A free, open-source system for microarray data management and analysis. Biotechniques 34: 374-378, 2003.

33. Livak KJ and Schmittgen TD: Analysis of relative gene expression data using real-time quantitative PCR and the 2(-Delta Delta C(T)) method. Methods 25: 402-408, 2001.

34. Gargiulo S, Gramanzini M, Megna R, Greco A, Albanese S, Manfredi $\mathrm{C}$ and Brunetti A: Evaluation of growth patterns and body composition in $\mathrm{C} 57 \mathrm{Bl} / 6 \mathrm{~J}$ mice using dual energy X-ray absorptiometry. Biomed Res Int 2014: 253067, 2014.

35. Yu FY, Xie CQ, Sun JT, Peng W and Huang XW: Overexpressed miR-145 inhibits osteoclastogenesis in RANKL-induced bone marrow-derived macrophages and ovariectomized mice by regulation of Smad3. Life Sci 202: 11-20, 2018.
36. Frith JE, Kusuma GD, Carthew J, Li F, Cloonan N, Gomez GA and Cooper-White JJ: Mechanically-sensitive miRNAs bias human mesenchymal stem cell fate via mTOR signalling. Nat Commun 9: 257, 2018.

37. Wan Y: Bone marrow mesenchymal stem cells: Fat on and blast off by FGF21. Int J Biochem Cell Biol 45: 546-549, 2013.

This work is licensed under a Creative Commons Attribution-NonCommercial-NoDerivatives 4.0 International (CC BY-NC-ND 4.0) License. 